\title{
AN UNORIENTED SKEIN EXACT TRIANGLE FOR KNOT FLOER HOMOLOGY
}

\author{
Ciprian Manolescu
}

\begin{abstract}
Given a crossing in a planar diagram of a link in the three-sphere, we show that the knot Floer homologies of the link and its two resolutions at that crossing are related by an exact triangle. As a consequence, we deduce that for any quasi-alternating link, the total rank of its knot Floer homology is equal to the determinant of the link.
\end{abstract}

\section{Introduction}

Given a link $L \subset S^{3}$, there are various interesting knot homology theories associated to $L$. One of them is Khovanov's reduced theory $\widetilde{K h}(L)$ from [3],[4], a bigraded vector space over $\mathbb{F}=\mathbb{Z} / 2 \mathbb{Z}$ whose Euler characteristic is the Jones polynomial. Other theories can be obtained from symplectic geometry: for example, one can consider $-\Sigma(L)$, the double cover of $S^{3}$ branched along $L$ (with the orientation reversed), and apply to it the Heegaard Floer homology $\widehat{H F}$ functor of Ozsváth and Szabó [11]. In a similar vein, a very useful theory is the knot Floer homology $\widehat{H F K}(L)$ of OzsváthSzabó and Rasmussen [10], [16]. In its simplest form, $\widehat{H F K}(L)$ is a bigraded vector space whose Euler characteristic is the Alexander polynomial. Knot Floer homology is known to detect the genus of a knot [9], as well as whether a knot is fibered [7]. There exists a refinement of $\widehat{H F K}$ called link Floer homology [12], which detects the Thurston norm of the link complement [15]. For the purposes of this paper, we will consider all the theories with coefficients in $\mathbb{F}=\mathbb{Z} / 2 \mathbb{Z}$, and usually ignore all their gradings.

The three theories mentioned above are rather different in origin. Khovanov's theory was developed out of a study of representation theory and categorification, and was defined in a purely combinatorial fashion to start with. On the other hand, the Floer homologies $\widehat{H F}(-\Sigma(L))$ and $\widehat{H F K}(L)$ were originally constructed using pseudolomorphic disks, and a combinatorial description of them has only recently been found $([6],[18])$. The ways in which these three theories are related to one another, however, are still not completely understood.

One connection between $\widetilde{K h}(L)$ and $\widehat{H F}(-\Sigma(L))$ was pointed out by Ozsváth and Szabó in [14]. They observed that both of these theories satisfy unoriented skein exact triangles. More precisely, consider three links $L, L_{0}$ and $L_{1}$ that admit planar diagrams differing from each other only at one crossing, where they look as in Figure 1. Then there is an exact triangle

$$
\mathcal{H}(L) \rightarrow \mathcal{H}\left(L_{0}\right) \rightarrow \mathcal{H}\left(L_{1}\right) \rightarrow \mathcal{H}(L),
$$

Received by the editors September 25, 2006. 


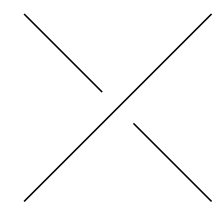

$L$

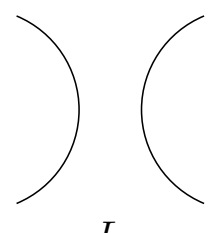

$L_{0}$

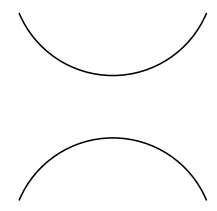

$L_{1}$

FiguRE 1. The links in the unoriented skein relation.

where the symbol $\mathcal{H}$ could stand for either $\widetilde{K h}$ or $\widehat{H F}(-\Sigma(\cdot))$. One can iterate this exact triangle by applying it to all crossings of $L$. Via some homological algebra, the result of this iteration process is a spectral sequence whose $E^{2}$ term is Khovanov homology, and which converges to $\widehat{H F}(-\Sigma(L))$. This implies an inequality of ranks:

$$
\mathrm{rk}_{\mathbb{F}} \widetilde{K h}(L) \geq \mathrm{rk}_{\mathbb{F}} \widehat{H F}(-\Sigma(L)) .
$$

In [14], Ozsváth and Szabó defined a class of links, called quasi-alternating links, for which (1) becomes equality and, furthermore, the ranks of the two theories are equal to $\operatorname{det}(L)$, the determinant of the link. In particular, all alternating links are quasi-alternating.

The main result of this paper is that knot Floer homology also has an unoriented skein exact triangle. Note that it was previously known to satisfy an oriented skein exact triangle, cf. [10].

Theorem 1. Let $L$ be a link in $S^{3}$. Given a planar diagram of $L$, let $L_{0}$ and $L_{1}$ be the two resolutions of $L$ at a crossing in that diagram, as in Figure 1. Denote by $l, l_{0}, l_{1}$ the number of components of the links $L, L_{0}$, and $L_{1}$, respectively, and set $m=\max \left\{l, l_{0}, l_{1}\right\}$. Then, there is an exact triangle

$\widehat{H F K}(L) \otimes V^{m-l} \rightarrow \widehat{H F K}\left(L_{0}\right) \otimes V^{m-l_{0}} \rightarrow \widehat{H F K}\left(L_{1}\right) \otimes V^{m-l_{1}} \rightarrow \widehat{H F K}(L) \otimes V^{m-l}$, where $V$ denotes a two-dimensional vector space over $\mathbb{F}$.

Ozsváth and Szabó [8], [12] proved that $\operatorname{rk}_{\mathbb{F}} \widehat{H F K}(L)=2^{l-1} \operatorname{det}(L)$ when $L$ is an alternating link with $l$ components. A simple consequence of Theorem 1 is a generalization of their result:

Corollary 2. If $L$ is a quasi-alternating link with $l$ components, then $\operatorname{rk}_{\mathbb{F}} \widehat{H F K}(L)=$ $2^{l-1} \cdot \operatorname{det}(L)$.

Rasmussen [17, Section 5] observed that $\widetilde{K h}$ and $\widehat{H F K}$ have equal ranks for many classes of knots (including most small knots), and asked for an explanation. Corollary 2 can be viewed as a partial answer to his question. Indeed, as we show in Section 5, many small knots are quasi-alternating.

As suggested in [17], a more convincing explanation for the similarities between $\widetilde{K h}$ and $\widetilde{H F K}$ (for knots) would be a spectral sequence whose $E^{2}$ term is $\widetilde{K h}$ and which converges to $\widehat{H F K}$. (For links of $l$ components, $\widetilde{K h}$ should be replaced with $\widetilde{K h} \otimes V^{l-1}$.) This would imply an inequality of ranks similar to (1), namely $2^{l-1}$. 
$\mathrm{rk}_{\mathbb{F}} \widetilde{K h} \geq \mathrm{rk}_{\mathbb{F}} \widehat{H F K}$. In turn, using the fact that $\widehat{H F K}$ detects the unknot [9], this would provide a positive answer to the following well-known conjecture:

Conjecture 3. If $K$ is a knot with $\operatorname{rk}_{\mathbb{F}} \widetilde{K h}(K)=1$, then $K$ is the unknot.

Unfortunately, Theorem 1 does not directly imply the inequality of ranks and hence Conjecture 3. One can iterate the unoriented skein triangle for $\widehat{H F K}$ and obtain a spectral sequence whose $E^{\infty}$ term is $\widehat{H F K} \otimes V^{n}$, for some large $n$. However, the presence of the $V$ factors makes it unclear whether the $E^{2}$ term of the spectral sequence is related to Khovanov homology. We leave this as an open problem.

We end this introduction with some remarks. First, the exact triangle from Theorem 1 is different from the other exact triangles in Floer homology, in that the maps do not even respect the homological gradings modulo 2. Also, the proof only works for the hat version of $H F K$, and it is not clear whether a similar triangle holds for the other versions. Finally, in this paper we only use the traditional definition of knot Floer homology, based on counting pseudo-holomorphic disks. It would be interesting to recover the same result, and perhaps do further computations, using the combinatorial definition of $[6]$ instead.

\section{Special Heegaard diagrams}

For the original definitions of knot Floer homology, we refer the reader to [10], [16], [12], [6]. Here we will use a special class of Heegaard diagrams, which are particular cases of the multiply-pointed diagrams defined in [6, Section2]. They are a variant of the Heegaard diagrams asssociated to knot projections in [8, Section 2].

Let $L \subset S^{3}$ be a link with $l$ components and $D$ a planar, connected projection of $L$. If $D$ has $c$ crossings, then it splits the plane into $c+2$ regions. Let $A_{0}$ denote the unbounded region in $\mathbb{R}^{2}-D$, and $A_{1}$ a region adjacent to $A$, separated from $A$ by an edge $e$. Denote the other regions by $A_{2}, \ldots, A_{c+1}$. Let $s_{1}, \ldots, s_{k}(k \geq l-1)$ be a collection of (not necessarily distinct) edges of $D$, chosen in such a way that for every component of the link, its projection contains at least one of the edges $s_{i}$ or $e$.

We denote by $\Sigma$ the boundary of a regular neighborhood of $D$ in $S^{3}$, a surface of genus $g=c+1$. To every region $A_{r}(r>0)$ we associate a curve $\alpha_{r}$ on $\Sigma$, following the boundary of $A_{r}$. To each crossing $v$ in $D$ we associate a curve $\beta_{v}$ on $\Sigma$ as indicated in Figure 2. Furthermore, we introduce an extra curve $\beta_{e}$ which is the meridian of the knot, supported in a neighborhood of the distinguished edge $e$. We also puncture the surface $\Sigma$ at two points on each side of $\beta_{e}$, as shown on the left side of Figure 3.

Finally, for every edge $s_{i}, i=1, \ldots, k$, we introduce a ladybug, i.e. an additional pair of alpha-beta curves on $\Sigma$, as well as an additional pair of punctures. This type of configuration is shown on the right side of Figure 3. The new beta curve $\beta_{s_{i}}$ is a meridian of the link, the two punctures lie on each of its two sides, and the new alpha curve $\alpha_{s_{i}}$ encircles the punctures.

In fact, we can think of the surface $\Sigma$ as being the union of several pieces, namely four-punctured spheres as in Figure 2 for each of the crossings, together with cylinders associated to $e$ and all the edges $s_{i}$ as in Figure 3. Note that there could be several cylinders for the same edge. The surface $\Sigma$, together with the collections of curves and punctures, is an example of a multi-pointed Heegaard diagram for $S^{3}$ compatible with $L$, in the sense of [6, Definition 2.1]. (However, unlike in the original sources, 

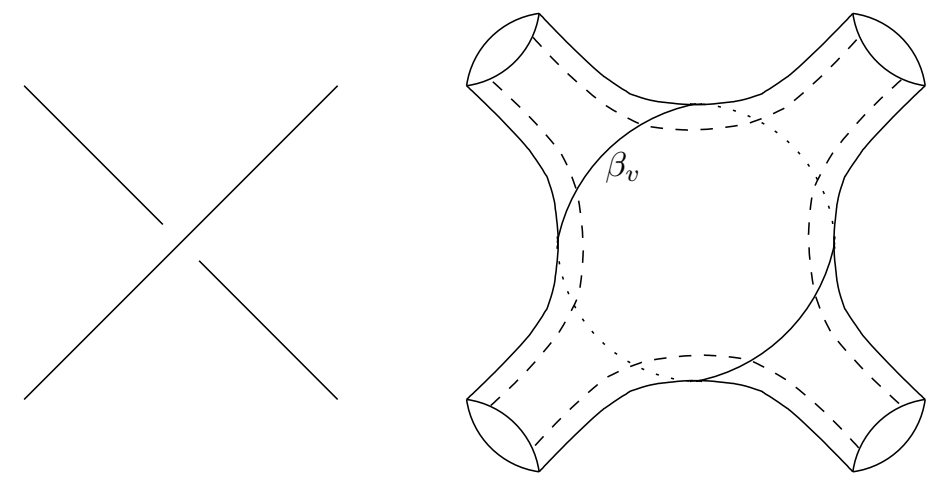

Figure 2. Given a crossing $v$ in the link diagram as on the left, we construct a piece of the Heegaard surface $\Sigma$ on the right. This piece contains four bits of alpha curves, shown in dashed lines, and one beta curve $\beta_{v}$.
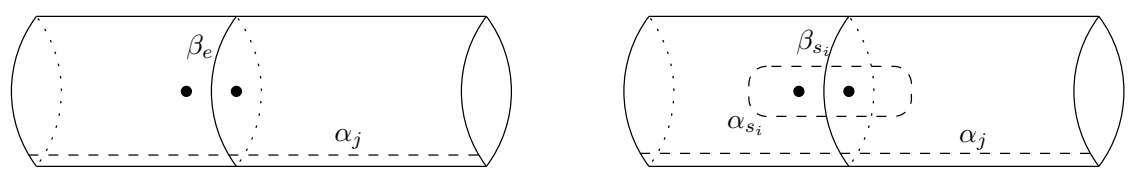

FIGURE 3. A neighborhood of the distinguished edge $e$ (left) and a ladybug around some edge $s_{i}$ (right).

here we are only interested in the hat version of $H F K$, and hence we do not need to distinguish between two different types of punctures.)

For the purpose of defining Floer homology, we need to ensure that the Heegaard diagram is admissible in the sense of [12, Definition 3.5]. This condition can be achieved by isotoping the curves, cf. [11], [12]. For example, one could stretch one tip of the alpha curve of each ladybug, and bring it close to the punctures associated to the distinguished edge $e$. It is easy to see that the result is an admissible diagram; see Figure 4 for an example. In general, we get a diagram with $k$ ladybugs, $g+k$ alpha curves, $g+k$ beta curves, and $2 k+2$ punctures in this diagram. We denote by $\widehat{\Sigma}$ the complement of the punctures in the surface $\Sigma$.

We then consider the torus $\mathbb{T}_{\alpha}$ which is the product of all the alpha curves and the torus $\mathbb{T}_{\beta}$ which is the product of all the beta curves. We view $\mathbb{T}_{\alpha}$ and $\mathbb{T}_{\beta}$ as totally real submanifolds of the symmetric product $\operatorname{Sym}^{g+k}(\widehat{\Sigma})$. Let $C F\left(\mathbb{T}_{\alpha}, \mathbb{T}_{\beta}\right)$ be the vector space freely generated by the intersection points between $\mathbb{T}_{\alpha}$ and $\mathbb{T}_{\beta}$. One endows $C F\left(\mathbb{T}_{\alpha}, \mathbb{T}_{\beta}\right)$ with the differential

$$
\partial \mathbf{x}=\sum_{\mathbf{y} \in \mathbb{T}_{\alpha} \cap \mathbb{T}_{\beta}} \sum_{\left\{\phi \in \pi_{2}(\mathbf{x}, \mathbf{y}) \mid \mu(\phi)=1\right\}} \#\left(\frac{\mathcal{M}(\phi)}{\mathbb{R}}\right) \mathbf{y} .
$$




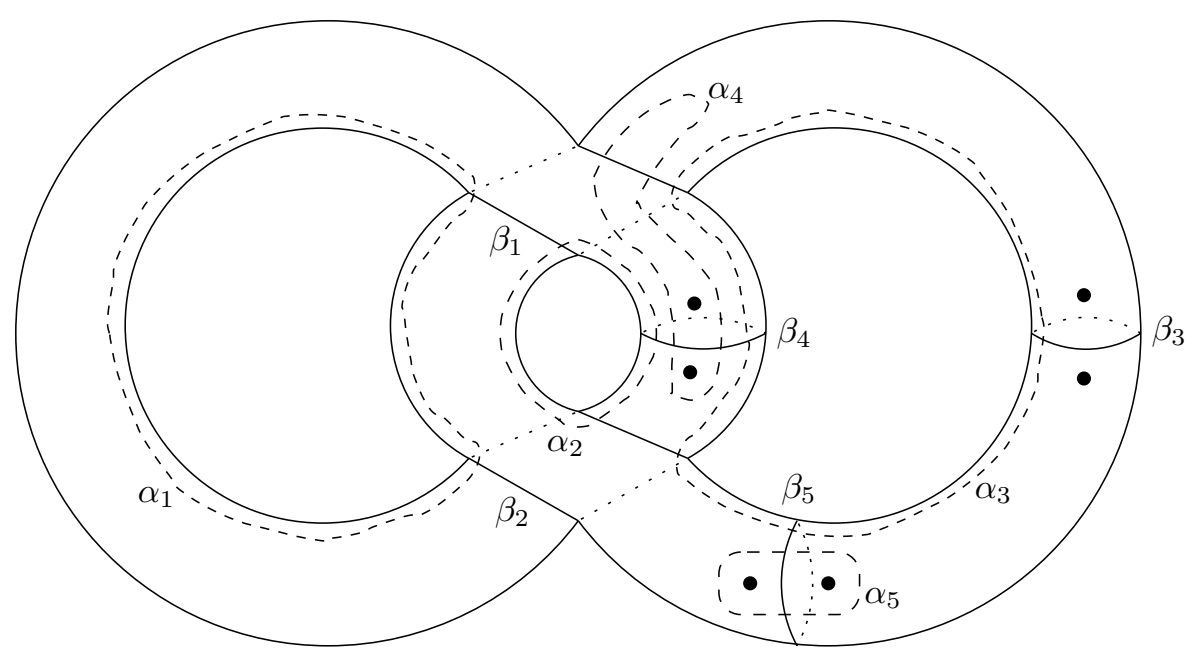

Figure 4. This is a special Heegaard diagram compatible with the Hopf link, with $g=3$ and $k=2$. The beta curves $\beta_{1}$ and $\beta_{2}$ are associated to the two crossings, $\beta_{3}$ to the distinguished edge, while $\beta_{4}$ and $\beta_{5}$ are each part of a ladybug. There are three alpha curves associated to planar bounded regions and two, $\alpha_{4}$ and $\alpha_{5}$, which are parts of ladybugs. One tip of $\alpha_{4}$ is stretched to achieve admissibility.

Here $\pi_{2}(\mathbf{x}, \mathbf{y})$ denotes the space of homology classes of Whitney disks connecting $\mathbf{x}$ to $\mathbf{y}$ in $\widehat{\Sigma}, \mathcal{M}(\phi)$ denotes the moduli space of pseudo-holomorphic representatives of $\phi$ (with respect to a suitable almost complex structure), and $\mu(\phi)$ denotes its formal dimension (Maslov index).

We can take the homology with respect to $\partial$, and obtain Floer homology groups $H F\left(\mathbb{T}_{\alpha}, \mathbb{T}_{\beta}\right)$. According to [6, Proposition 2.4], these are (up to a factor) the knot Floer homology groups of [10] and [16]:

$$
H F\left(\mathbb{T}_{\alpha}, \mathbb{T}_{\beta}\right) \cong \widehat{H F K}(L) \otimes V^{k-l} .
$$

\section{Proof of Theorem 1}

Consider three links $L, L_{0}, L_{1}$, with planar diagrams $D, D_{0}, D_{1}$ differing from each other as in the statement of Theorem 1. Among them there is exactly one which has $m$ components, while the other two have only $m-1$ components. Without loss of generality, let us assume that $L$ has $m$ components. (The other two cases are similar.)

Pick a special Heegaard diagram for $L$ as in Section 2. We choose it to have the minimum possible number of ladybugs, namely $m-1$. We denote the alpha and beta curves in the diagram by $\alpha_{i}$ and $\beta_{i}$, respectively, with $i=1, \ldots, n$, where $n=g+m-1$. We reserve the index $n$ for the beta curve $\beta=\beta_{n}$ associated to the particular crossing $v$ where $D$ differs from $D_{0}$ and $D_{1}$.

Let us call $\mathcal{P}$ the piece of the Heegaard diagram corresponding to the crossing $v$ as in Figure 2. Topologically, $\mathcal{P}$ is a sphere with four disks removed. In $\mathcal{P}$, let us replace $\beta$ by the curve $\gamma$ pictured on the left side of Figure 5 . It can be checked directly that 


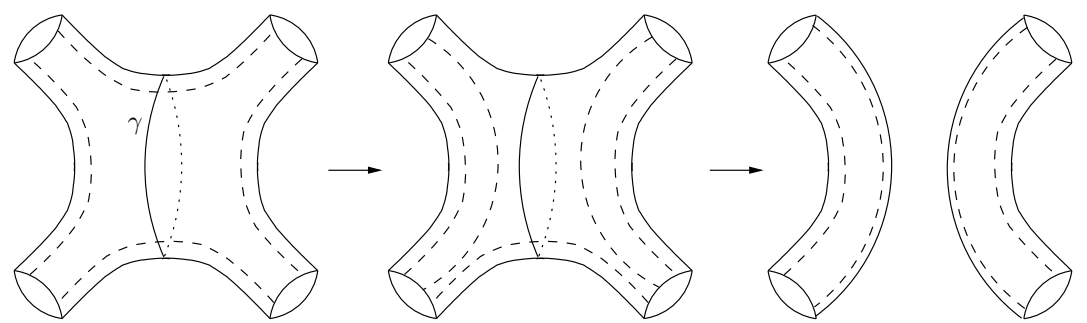

Figure 5. A handleslide of the alpha curves, then a de-stabilization preceded by suitable handleslides of beta curves over $\gamma$. The result is a special Heegaard diagram for the resolution $L_{0}$.

this gives a multi-pointed Heegaard diagram for the resolution $L_{0}$. Alternatively, one can perform the moves shown in Figure 5 to arrive at a special Heegaard diagram for $L_{0}$, of the type considered in the previous section.

A similar construction can be used to obtain a Heegaard diagram for $L_{1}$ : instead of the vertical curve $\gamma$ in $\mathcal{P}$, we need to consider a horizontal curve $\delta$, separating the two upper boundaries of $\mathcal{P}$ from the two lower boundaries. If we identify $S^{2}$ with the plane together with a point at infinty, and $\mathcal{P}$ with the complement of four disks in $S^{2}$, then the curves $\beta, \gamma$, and $\delta$ are arranged as in Figure 6 . Their intersection points are:

$$
\beta \cap \gamma=\{A, U\}, \quad \gamma \cap \delta=\{B, V\}, \quad \delta \cap \beta=\{C, W\} .
$$

Consider also a small perturbation $\beta^{\prime}$ of the curve $\beta$, such that the two intersect in two points. We denote by $C^{\prime}$ and $W^{\prime}$ the intersection points of $\beta^{\prime}$ and $\delta$ which are close to $C$ and $W$, respectively. Furthermore, for each $i=1, \ldots, n-1$, we choose small perturbations $\gamma_{i}, \delta_{i}, \beta_{i}^{\prime}$ of the curve $\beta_{i}$, such that (for a fixed $i$ ) each intersects $\beta_{i}$ in two points, and any two of them intersect each other in two points as well. In general, if $\eta$ and $\eta^{\prime}$ are (homologically nontrivial) isotopic curves in the punctured surface $\hat{\Sigma}$ intersecting in two points, then the Floer chain complex $C F\left(\eta, \eta^{\prime}\right)$ has rank two, being generated by the intersection points. We denote by $M_{\eta \eta^{\prime}}$ the point which gives the top degree generator of $C F\left(\eta, \eta^{\prime}\right)$, and by $M_{\eta^{\prime} \eta}$ the other intersection point. For example, $\beta$ and $\beta^{\prime}$ intersect in the points $M_{\beta^{\prime} \beta}$ and $M_{\beta \beta^{\prime}}$ shown in Figure 6.

Set $\mathbb{T}_{\alpha}=\alpha_{1} \times \cdots \times \alpha_{n}$ and

$$
\begin{aligned}
& \mathbb{T}_{\beta}=\beta_{1} \times \cdots \times \beta_{n-1} \times \beta ; \quad \mathbb{T}_{\gamma}=\gamma_{1} \times \cdots \times \gamma_{n-1} \times \gamma ; \\
& \mathbb{T}_{\delta}=\delta_{1} \times \cdots \times \delta_{n-1} \times \delta ; \quad \mathbb{T}_{\beta^{\prime}}=\beta_{1}^{\prime} \times \cdots \times \beta_{n-1}^{\prime} \times \beta^{\prime} .
\end{aligned}
$$

Viewing them as totally real tori in $\operatorname{Sym}^{n}(\widehat{\Sigma})$, we have

$$
\begin{gathered}
H F\left(\mathbb{T}_{\alpha}, \mathbb{T}_{\beta}\right)=H F\left(\mathbb{T}_{\alpha}, \mathbb{T}_{\beta^{\prime}}\right)=\widehat{H F K}(L) ; \\
H F\left(\mathbb{T}_{\alpha}, \mathbb{T}_{\gamma}\right)=\widehat{H F K}\left(L_{0}\right) \otimes V ; H F\left(\mathbb{T}_{\alpha}, \mathbb{T}_{\delta}\right)=\widehat{H F K}\left(L_{1}\right) \otimes V .
\end{gathered}
$$

Thus, the exact triangle from the statement of Theorem 1 can be written as:

$$
\ldots \rightarrow H F\left(\mathbb{T}_{\alpha}, \mathbb{T}_{\beta}\right) \rightarrow H F\left(\mathbb{T}_{\alpha}, \mathbb{T}_{\gamma}\right) \rightarrow H F\left(\mathbb{T}_{\alpha}, \mathbb{T}_{\delta}\right) \rightarrow \ldots
$$




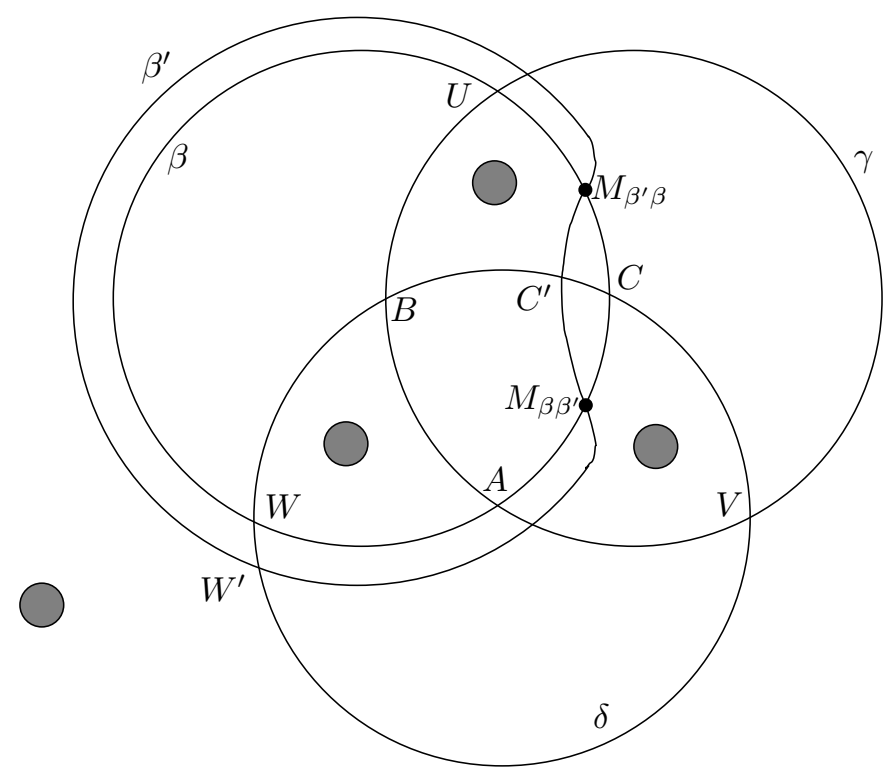

FiguRE 6 . We show various curves in the four-punctured sphere $\mathcal{P}$. The four gray disks correspond to tubes which link $\mathcal{P}$ to the rest of the Heegaard surface $\Sigma$.

The strategy for proving (2) is the same as the one used by Ozsvath and Szabó in [14] for double-branched covers. Roughly, the principle behind the proof is the following: "If the triangle counts are zero, and the quadrilateral counts are one (modulo two), then the exact triangle holds true."

To make this precise, we need the following input from homological algebra, which was also used in [8] and [5]:

Lemma 4. Let $\left\{\left(C_{k}, \partial_{k}\right)\right\}_{k \in \mathbb{Z} / 3 \mathbb{Z}}$ be a collection of chain complexes over $\mathbb{F}=\mathbb{Z} / 2 \mathbb{Z}$

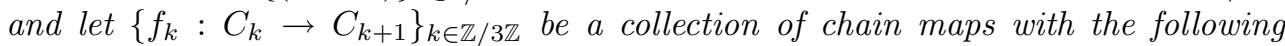
properties:

(1) The composite $f_{k+1} \circ f_{k}: C_{k} \rightarrow C_{k+2}$ is chain-homotopic to zero, by a chain homotopy $H_{k}$ :

$$
\partial_{k+2} \circ H_{k}+H_{k} \circ \partial_{k}=f_{k+1} \circ f_{k} ;
$$

(2) The sum

$$
\psi_{k}=f_{k+2} \circ H_{k}+H_{k+1} \circ f_{k}: C_{k} \rightarrow C_{k}
$$

(which is a chain map) induces an isomorphism on homology.

Then the sequence

$$
\cdots \longrightarrow H_{*}\left(C_{k-1}\right) \stackrel{\left(f_{k-1}\right)_{*}}{\longrightarrow} H_{*}\left(C_{k}\right) \stackrel{\left(f_{k}\right)_{*}}{\longrightarrow} H_{*}\left(C_{k+1}\right) \longrightarrow \cdots
$$

is exact. 
In our situation, we seek to apply Lemma 4 to

$$
C_{0}=C F\left(\mathbb{T}_{\alpha}, \mathbb{T}_{\beta}\right), \quad C_{1}=C F\left(\mathbb{T}_{\alpha}, \mathbb{T}_{\gamma}\right), \quad C_{2}=C F\left(\mathbb{T}_{\alpha}, \mathbb{T}_{\delta}\right)
$$

The maps $f_{k}$ and $H_{k}$ will be particular examples of the following well-known natural maps in Floer homology, cf. [1], [2]. For Lagrangians $T_{0}, T_{1}, \ldots, T_{s}$ in a symplectic manifold $M$ (with good topological and analytic properties, e.g. transversality, no bubbling), the choice of a compatible almost complex structure produces maps

$$
\begin{gathered}
F_{T_{0}, \ldots, T_{s}}: \bigotimes_{i=1}^{s} C F\left(T_{i-1}, T_{i}\right) \rightarrow C F\left(T_{0}, T_{s}\right) \\
F_{T_{0}, \ldots, T_{s}}\left(\mathbf{x}_{1} \otimes \cdots \otimes \mathbf{x}_{s}\right)=\sum_{\mathbf{y} \in T_{0} \cap T_{s}\left\{\phi \in \pi_{2}\left(\mathbf{x}_{1}, \ldots, \mathbf{x}_{s}, \mathbf{y}\right) \mid \mu(\phi)=0\right\}}(\# \mathcal{M}(\phi)) \cdot \mathbf{y} .
\end{gathered}
$$

Here $\pi_{2}\left(\mathbf{x}_{1}, \ldots, \mathbf{x}_{s}, \mathbf{y}\right)$ denotes the set of homotopy classes of Whitney $(s+1)$-gons in $M$, with boundaries on $T_{0}, \ldots, T_{s}$ and vertices $\mathbf{x}_{1}, \ldots, \mathbf{x}_{s}, \mathbf{y}$; also, $\mu(\phi)$ is the Maslov index, and $\mathcal{M}(\phi)$ is the moduli space of pseudo-holomorphic representatives of $\phi$. (When $s=1$, we ask for $\mu(\phi)=1$ instead of 0 , and divide out the moduli space by the automorphism group $\mathbb{R}$ before counting. The map $F_{T_{0}, T_{1}}$ is then the differential in the Floer complex.) These maps satisfy the generalized associativity relations:

$$
\sum_{0 \leq i<j \leq s} F_{T_{0}, \ldots, T_{i-1}, T_{i}, T_{j}, \ldots, T_{s}} \circ F_{T_{i}, \ldots, T_{j}}=0 .
$$

The maps $F_{T_{0}, \ldots, T_{s}}$ are also well-defined, and satisfy (3), when the $T_{i}$ 's are totallyreal product tori in $\operatorname{Sym}^{n}(\widehat{\Sigma})$, cf. [11], [14, Section 4.2].

Before applying these maps in our setting, let us introduce some more notation. Given one of the intersection points shown in Figure 6, for example $A \in \beta \cap \gamma$, we obtain a corresponding generator in the corresponding Floer chain complex $C F\left(\mathbb{T}_{\beta}, \mathbb{T}_{\gamma}\right)$ by adjoining to $A$ the top degree intersection points $M_{\beta_{i} \gamma_{i}} \in \beta_{i} \cap \gamma_{i}$. We denote the resulting generator by the respective lowercase letter in bold; for example:

$$
\begin{gathered}
\mathbf{a}=M_{\beta_{1} \gamma_{1}} \times M_{\beta_{2} \gamma_{2}} \times \cdots \times M_{\beta_{n-1} \gamma_{n-1}} \times A \in C F\left(\mathbb{T}_{\beta}, \mathbb{T}_{\gamma}\right) \\
\mathbf{u}=M_{\beta_{1} \gamma_{1}} \times M_{\beta_{2} \gamma_{2}} \times \cdots \times M_{\beta_{n-1} \gamma_{n-1}} \times U \in C F\left(\mathbb{T}_{\beta}, \mathbb{T}_{\gamma}\right) \\
\mathbf{b}=M_{\gamma_{1} \delta_{1}} \times M_{\gamma_{2} \delta_{2}} \times \cdots \times M_{\gamma_{n-1} \delta_{n-1}} \times B \in C F\left(\mathbb{T}_{\gamma}, \mathbb{T}_{\delta}\right), \text { etc. }
\end{gathered}
$$

We can now define the maps needed in Lemma 4 . We choose

$$
\begin{aligned}
& f_{0}: C F\left(\mathbb{T}_{\alpha}, \mathbb{T}_{\beta}\right) \rightarrow C F\left(\mathbb{T}_{\alpha}, \mathbb{T}_{\gamma}\right), f_{0}(\mathbf{x})=F_{\mathbb{T}_{\alpha}, \mathbb{T}_{\beta}, \mathbb{T}_{\gamma}}(\mathbf{x} \otimes(\mathbf{a}+\mathbf{u})) ;
\end{aligned}
$$

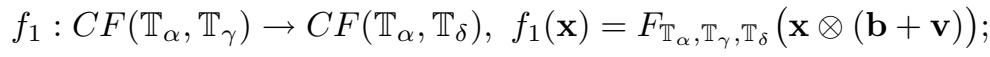

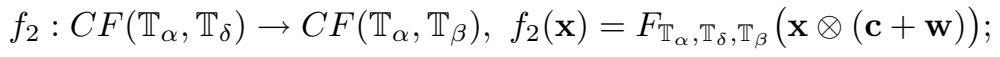

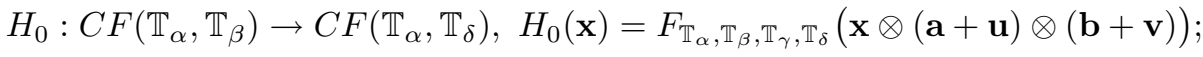

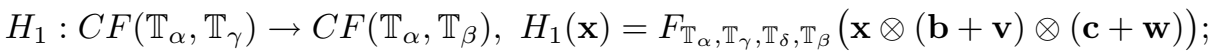

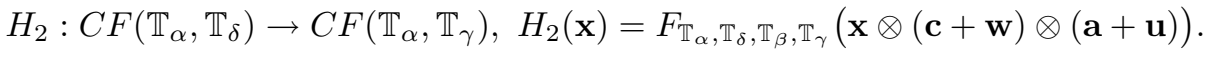

Lemma 5. The maps $f_{k}(k \in \mathbb{Z} / 3 \mathbb{Z})$ are chain maps. 
Proof. Equation (3) for $s=2$ says that if $\mathbf{a}+\mathbf{u}$ is a cycle in $C F\left(\mathbb{T}_{\beta}, \mathbb{T}_{\gamma}\right)$, then the map $f_{0}$ commutes with the Floer differentials. We claim that both a and $\mathbf{u}$ are cycles. Note that if $u$ is a pseudo-holomorphic disk connecting a to some other intersection point $\mathbf{y} \in \mathbb{T}_{\beta} \cap \mathbb{T}_{\gamma}$, then $\mathbf{y}$ is an $n$-tuple of points, one of which is $y_{n} \in\{A, U\}$. Let $\mathcal{D}(u)$ be the domain in $\Sigma$ associated to $u$, cf. [11, Definition 2.13].

Observe that if we delete from $\Sigma$ the $\beta$ and $\gamma$ curves, the only connected componets which do not contain punctures are the pairs of thin bigons joining $M_{\beta_{i} \gamma_{i}}$ to $M_{\gamma_{i} \beta_{i}}$, for each $i=1, \ldots, n-1$. Since $u$ is a pseudo-holomorphic disk in $\operatorname{Sym}^{n}(\widehat{\Sigma})$, the domain $\mathcal{D}(u)$ cannot go over a puncture, hence it is a sum of those thin bigons (with some multiplicities). In particular, $y_{n}$ must be $A$.

If we impose the condition on $u$ to have Maslov index one, then $\mathcal{D}(u)$ is exactly one thin bigon. Each bigon has a holomorphic representative, hence contributes a term to the differential of $\mathbf{a}$. However, the bigons come in pairs, which means that $\partial \mathbf{a}=0$. Similarly, $\partial \mathbf{u}=0$. The cases of $f_{1}$ and $f_{2}$ are completely analogous.

Lemma 6. The maps $f_{k}$ and $H_{k}$ satisfy condition (1) in Lemma 4.

Proof. We claim that

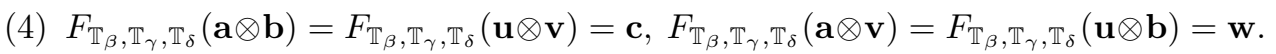

This follows from an inspection of the $\alpha \beta \gamma$ triangles in Figure 6 , together with the observation that the domains of pseudo-holomorphic triangles cannot go over the tubes, by an argument similar to the one in the proof of Lemma 5 .

Summing up the relations in (4) we get:

$$
F_{\mathbb{T}_{\beta}, \mathbb{T}_{\gamma}, \mathbb{T}_{\delta}}((\mathbf{a}+\mathbf{u}) \otimes(\mathbf{b}+\mathbf{v}))=0
$$

Using the associativity relation (3) in the case $s=3$, together with (5) and the fact that $\mathbf{a}+\mathbf{u}$ and $\mathbf{b}+\mathbf{v}$ are cycles, we obtain $\partial_{1} \circ H_{0}+H_{0} \circ \partial_{0}=f_{1} \circ f_{0}$. The cases $k=1$ and $k=2$ are similar.

Lemma 7. The maps $f_{k}$ and $H_{k}$ satisfy condition (2) in Lemma 4.

Proof. We will prove condition (2) for $k=0$, as the other cases are completely analogous. Let

$$
\mathbf{m}=M_{\beta_{1} \beta_{1}^{\prime}} \times \cdots \times M_{\beta_{n-1} \beta_{n-1}^{\prime}} \times M_{\beta \beta^{\prime}} \in C F\left(\mathbb{T}_{\beta}, \mathbb{T}_{\beta^{\prime}}\right)
$$

The map

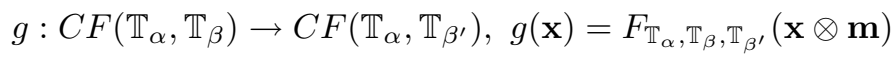

is readily seen to be an isomorphism on homology. Observe also that the maps $g \circ f_{2}$ and $g \circ H_{1}$ are homotopy equivalent to $f_{2}^{\prime}$ and $H_{1}^{\prime}$, respectively, where

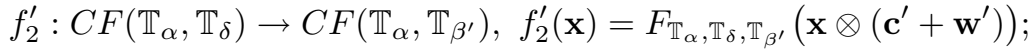

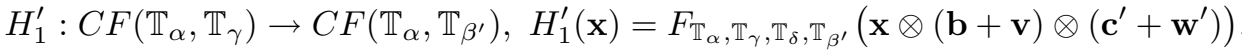

Therefore, the statement that $f_{2} \circ H_{0}+H_{1} \circ f_{0}$ is an isomorphism on homology is equivalent to

$$
g_{1}=f_{2}^{\prime} \circ H_{0}+H_{1}^{\prime} \circ f_{0}: C F\left(\mathbb{T}_{\alpha}, \mathbb{T}_{\beta}\right) \rightarrow C F\left(\mathbb{T}_{\alpha}, \mathbb{T}_{\beta^{\prime}}\right)
$$


being an isomorphism on homology.

Consider the map

$$
g_{2}: C F\left(\mathbb{T}_{\alpha}, \mathbb{T}_{\beta}\right) \rightarrow C F\left(\mathbb{T}_{\alpha}, \mathbb{T}_{\beta^{\prime}}\right), g_{2}(\mathbf{x})=F_{\mathbb{T}_{\alpha}, \mathbb{T}_{\beta}, \mathbb{T}_{\beta^{\prime}}}(\mathbf{x} \otimes \theta),
$$

where

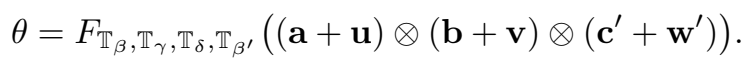

Finally, define $H: C F\left(\mathbb{T}_{\alpha}, \mathbb{T}_{\beta}\right) \rightarrow C F\left(\mathbb{T}_{\alpha}, \mathbb{T}_{\beta^{\prime}}\right)$ by

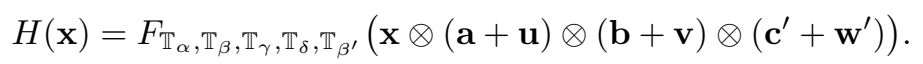

Let us apply the $s=4$ version of the associativity relation (3) to the tori $\mathbb{T}_{\alpha}, \mathbb{T}_{\beta}, \mathbb{T}_{\gamma}$, $\mathbb{T}_{\delta}, \mathbb{T}_{\beta^{\prime}}$ (in this order), evaluating all the summands at an element of the form $\mathbf{x} \otimes$ $(\mathbf{a}+\mathbf{u}) \otimes(\mathbf{b}+\mathbf{v}) \otimes\left(\mathbf{c}^{\prime}+\mathbf{w}^{\prime}\right)$. We get ten summands; three of them evaluate to zero because $\mathbf{a}+\mathbf{u}, \mathbf{b}+\mathbf{v}$ and $\mathbf{c}^{\prime}+\mathbf{w}^{\prime}$ are cycles, cf. Lemma 5 ; two others evaluate to zero because

$$
F_{\mathbb{T}_{\beta}, \mathbb{T}_{\gamma}, \mathbb{T}_{\delta}}((\mathbf{a}+\mathbf{u}) \otimes(\mathbf{b}+\mathbf{v}))=F_{\mathbb{T}_{\gamma}, \mathbb{T}_{\beta}, \mathbb{T}_{\beta^{\prime}}}\left((\mathbf{b}+\mathbf{v}) \otimes\left(\mathbf{c}^{\prime}+\mathbf{w}^{\prime}\right)\right)=0,
$$

cf. Lemma 6 . The remaining five summands give the relation:

$$
g_{1}(\mathbf{x})+g_{2}(\mathbf{x})+(\partial \circ H)(\mathbf{x})+(H \circ \partial)(\mathbf{x})=0 .
$$

Therefore, $g_{1}$ and $g_{2}$ are chain homotopic. It suffices now to show that $g_{2}$ is an isomorphism on homology. At this time we need to make use of the homological (Maslov) grading on Floer complexes. We claim that

$$
\theta=\mathbf{m}+\text { (lower degree terms). }
$$

In other words, the claim is that the count of Maslov index zero, pseudo-holomorphic quadrilaterals having as vertices one of $\mathbf{a}$ and $\mathbf{u}$, one of $\mathbf{b}$ and $\mathbf{v}$, one of $\mathbf{c}^{\prime}$ and $\mathbf{w}^{\prime}$, as well as $\mathbf{m}$, is odd. This follows by inspecting the corresponding quadrilaterals in Figure 6, and coupling them with quadrilaterals between $\beta_{i}, \gamma_{i}, \delta_{i}$ and $\beta_{i}^{\prime}(i=$ $1, \ldots, n-1)$, as in the proof of Theorem 4.5 in [14]. With the perturbation $\beta^{\prime}$ of $\beta$ being chosen exactly as in Figure 6, there is a unique useful (Maslov index zero) quadrilateral there, the one with vertices $A, B, C^{\prime}$ and $M_{\beta \beta^{\prime}}$ which is positioned right in the middle of the picture.

Equation (6) implies that $g_{2}=g+$ (lower degree terms), hence $g_{2}$ is an isomorphism on homology.

The proof of Theorem 1 is completed by putting together Lemmas 4, 5, 6 and 7 .

\section{An example}

We illustrate the unoriented skein exact triangle in the case of the links in Figure 7. The corresponding Heegaard diagrams are shown in Figure 8. That picture can be simplified by handlesliding the curves $\beta$ and $\gamma$ over $\beta_{1}$, handlesliding $\beta$ and $\delta$ over $\beta_{2}$, and then de-stabilizing the pairs $\left(\alpha_{1}, \beta_{1}\right)$ and $\left(\alpha_{2}, \beta_{2}\right)$.

The result is shown in Figure 9. The curve $\alpha=\alpha_{3}$ intersects each of the curves $\beta$, $\gamma$ and $\delta$ in two points, so that each of the corresponding Floer homology groups are two-dimensional. The triangle (2) takes the form

$$
H F(\alpha, \beta) \stackrel{f_{0}}{\longrightarrow} H F(\alpha, \gamma) \stackrel{f_{1}}{\longrightarrow} H F(\alpha, \delta) \stackrel{f_{2}}{\longrightarrow} H F(\alpha, \beta) .
$$



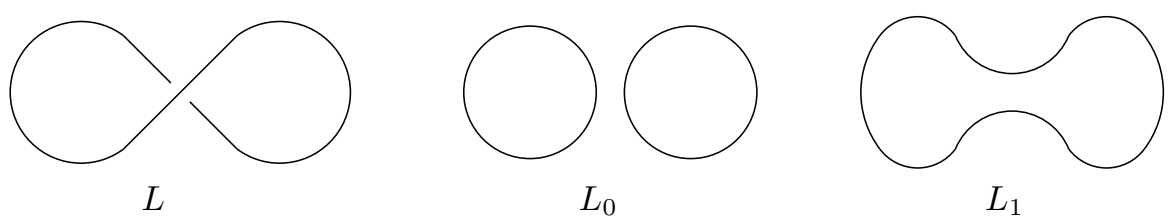

Figure 7. A diagram of the unknot and its resolutions.

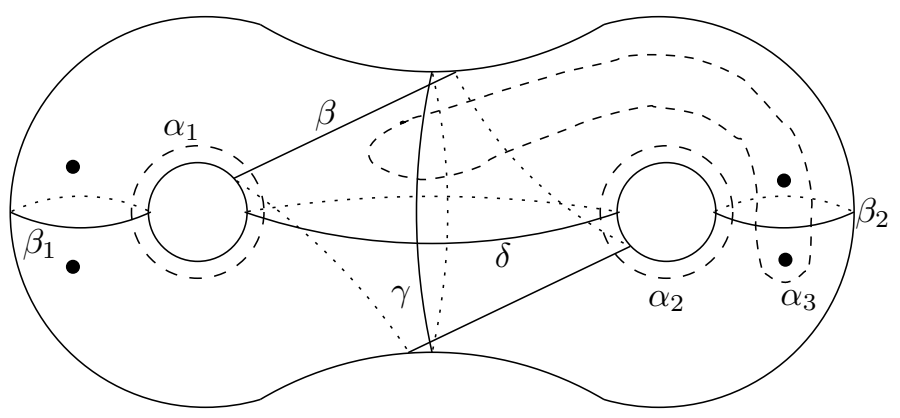

Figure 8 . The triples of curves $\left(\alpha_{1}, \alpha_{2}, \alpha_{3}\right)$ and $\left(\beta_{1}, \beta_{2}, \beta\right)$ give a Heegaard diagram for $L$. To obtain Heegaard diagrams for $L_{0}$ and $L_{1}$, replace the curve $\beta$ by $\gamma$ and $\delta$, respectively.

The maps can be computed by inspecting the triangles in Figure 9. For example, the $\alpha \delta \beta$ triangle with vertices $S, W$ and $N$ produces a summand of $N$ in $f_{2}(S)$. We obtain:

$f_{0}(M)=Q ; f_{0}(N)=Q ; f_{1}(P)=R+S ; f_{1}(Q)=0 ; f_{2}(R)=M+N ; f_{2}(S)=M+N$.

\section{Quasi-alternating knots}

Let us recall the definition of the class $\mathcal{Q}$ of quasi-alternating links from [14]. The set $\mathcal{Q}$ is the smallest set of links satisfying the following properties:

- The unknot is in $\mathcal{Q}$;

- If $L$ is a link which admits a projection with a crossing such that

(1) Both resolutions $L_{0}, L_{1} \in \mathcal{Q}$,

(2) $\operatorname{det}(L)=\operatorname{det}\left(L_{0}\right)+\operatorname{det}\left(L_{1}\right)$,

then $L$ is in $\mathcal{Q}$.

Note that if $L \in \mathcal{Q}$, then $\operatorname{det}(L) \geq 1$, with equality if and only if $L$ is the unknot.

Proof of Corollary 2. For any link $L$, if we use the bigradings on $\widehat{H F K}(L)$ introduced in [10], [16], then the Euler characteristic of $\widehat{H F K}(L)$ is the Alexander polynomial $\left(t^{-1 / 2}-t^{1 / 2}\right)^{l-1} \Delta_{L}(t)$. The determinant of the link is $\operatorname{det}(L)=\left|\Delta_{L}(-1)\right|$, hence for every link we have:

$$
2^{l-1} \cdot \operatorname{det}(L) \leq \operatorname{rk}_{\mathbb{F}} \widehat{H F K}(L) .
$$




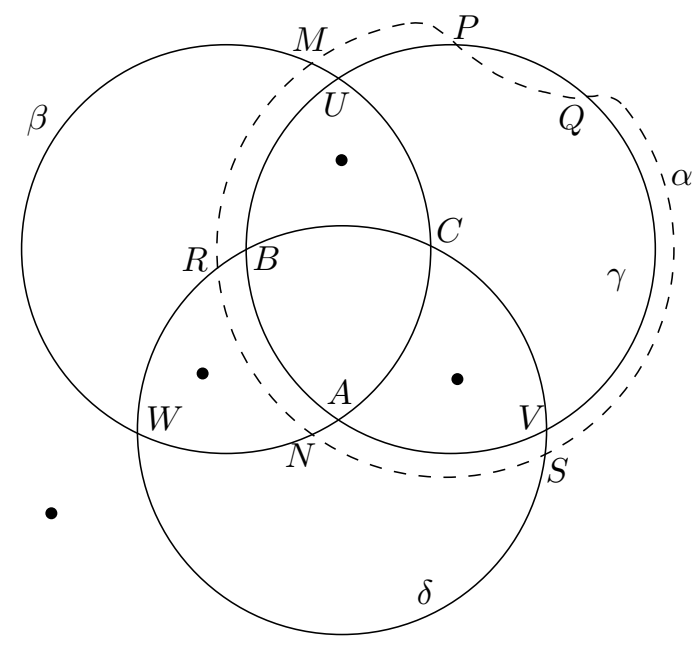

Figure 9. Figure 8 after two de-stabilizations.

We prove by induction on $\operatorname{det}(L)$ that a quasi-alternating $\operatorname{link} L$ satisfies the inequality in the other direction, $\operatorname{rk}_{\mathbb{F}} \widehat{H F K}(L) \leq 2^{l-1} \cdot \operatorname{det}(L)$. Indeed, for the unknot we have $\operatorname{rk}_{\mathbb{F}} \widehat{H F K}=1$, and then the inductive step follows readily from the definition of $\mathcal{Q}$.

We note that quasi-alternating knots are very frequent among small knots. Indeed, all but eleven of the prime knots with nine or less crossings are alternating, and alternating knots are quasi-alternating by [14, Lemma 3.2]. Furthermore, eight of the eleven non-alternating knots can be checked to be quasi-alternating; see Figure 10. This leaves only the knots $8_{19}$ and $9_{42}$, which have $\operatorname{rk}_{\mathbb{F}} \widehat{H F K}(K)>\operatorname{det}(K)$ by [10], [13], and hence cannot be quasi-alternating, as well as $8_{20}$, which the author does not know if it is quasi-alternating. 1

\section{Acknowledgements}

I owe a great debt of gratitude to Peter Ozsváth; his suggestions and encouragement have been essential in completing this paper. I am also grateful to Nathan Dunfield and Jacob Rasmussen for several very helpful discussions, and to John Baldwin for pointing out a few minor errors in a previous version.

This work was completed while I was a Clay Research Fellow; I would like to thank the Clay Mathematical Institute for its generous support.

\section{References}

[1] V. deSilva, Products in the symplectic Floer homology of Lagrangian intersections, Ph.D. thesis, Oxford University (1999).

[2] K. Fukaya, Y.-G. Oh, K. Ono, and H. Ohta, Lagrangian intersection Floer theory-anomaly and obstruction, Kyoto University (2000).

\footnotetext{
${ }^{1}$ Note added in proof: John Baldwin informed the author that $8_{20}$ is quasi-alternating as well.
} 

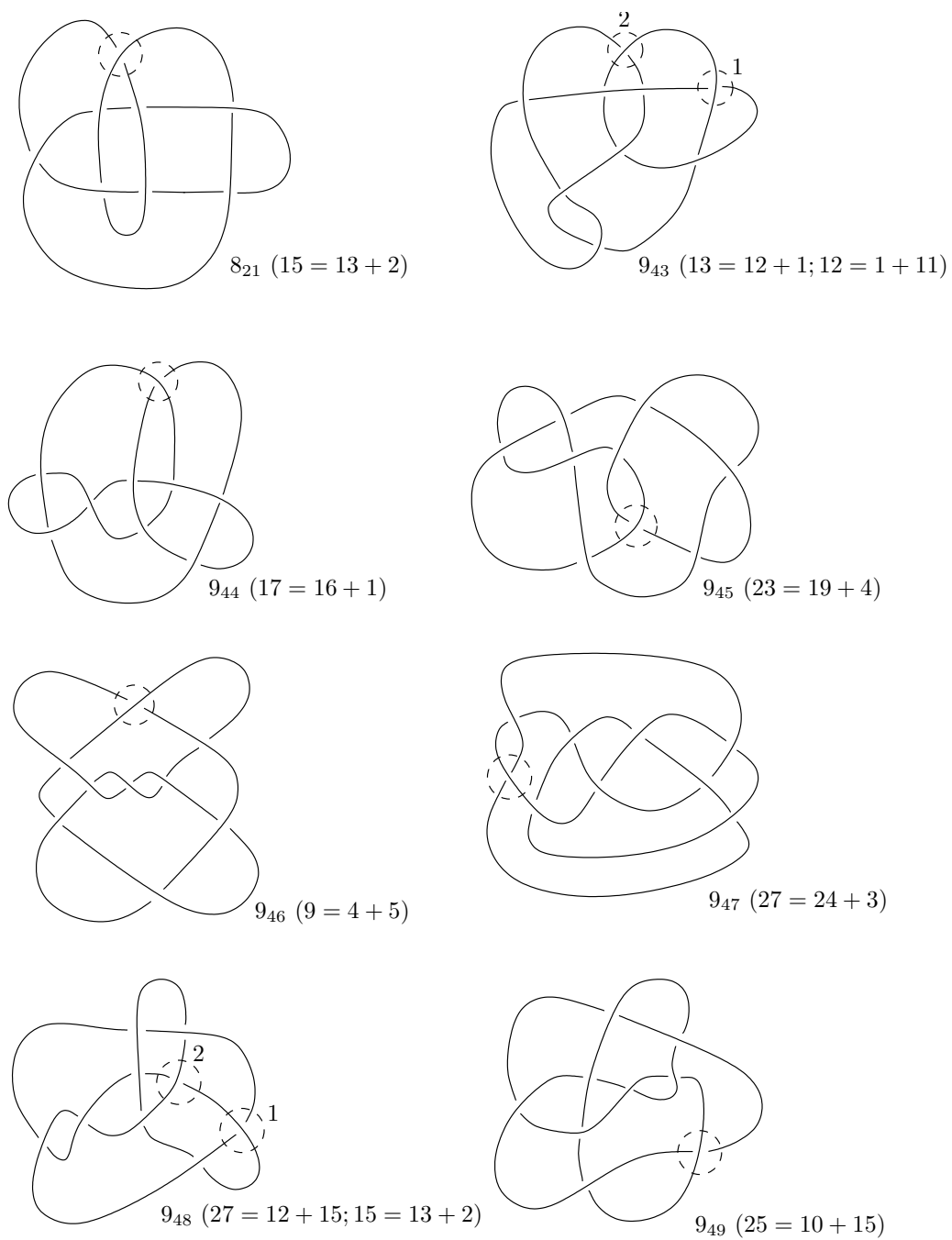

FiguRE 10. Quasi-alternating knots with at most nine crossings that are not alternating. Six of them can be resolved at one crossing to produce two alternating links; we write the relation $\operatorname{det}(L)=$ $\operatorname{det}\left(L_{0}\right)+\operatorname{det}\left(L_{1}\right)$ in parantheses. For the knots $9_{43}$ and $9_{48}$, one of the resolutions at the crossing numbered 1 is alternating and the other is not; however, if we resolve the non-alternating one at the crossing numbered 2 , we obtain two alternating links.

[3] M. Khovanov, A categorification of the Jones polynomial, Duke Math. J. 101 (2000), no. 3, 359-426.

[4] - Patterns in knot cohomology. I, Experiment. Math. 12 (2003), no. 3, 365-374.

[5] P. B. Kronheimer, T. S. Mrowka, P. S. Ozsváth, and Z. Szabó, Monopoles and lens space surgeries, Ann. of Math. (2) 165 (2007), no. 2, 457-546. 
[6] C. Manolescu, P. S. Ozsváth, and S. Sarkar, A combinatorial description of knot Floer homology (2006). Preprint, math.GT/0607691.

[7] Y. Ni, Knot Floer homology detects fibred knots (2006). Preprint, math.GT/0607156.

[8] P. Ozsváth and Z. Szabó, Heegaard Floer homology and alternating knots, Geom. Topol. 7 (2003) 225-254 (electronic).

[9] - Holomorphic disks and genus bounds, Geom. Topol. 8 (2004) 311-334 (electronic).

[10] - Holomorphic disks and knot invariants, Adv. Math. 186 (2004), no. 1, 58-116.

[11] - Holomorphic disks and three-manifold invariants: properties and applications, Ann. of Math. (2) 159 (2004), no. 3, 1159-1245.

[12] - Holomorphic disks and link invariants (2005). Preprint, math.GT/0512286.

[13] - On knot Floer homology and lens space surgeries, Topology 44 (2005), no. 6, 1281-1300.

[14] - On the Heegaard Floer homology of branched double-covers, Adv. Math. 194 (2005), no. $1,1-33$.

[15] - Link Floer homology and the Thurston norm (2006). Preprint, math.GT/0601618.

[16] J. Rasmussen, Floer homology and knot complements, Ph.D. thesis, Harvard University (2003). Preprint, math.GT/0306378.

[17] - Knot polynomials and knot homologies, in Geometry and topology of manifolds, Vol. 47 of Fields Inst. Commun., 261-280, Amer. Math. Soc., Providence, RI (2005).

[18] S. Sarkar and J. Wang, A combinatorial description of some Heegaard Floer homologies (2006). Preprint, math.GT/0607777.

Department of Mathematics, Columbia University, 2990 Broadway, MC 4410, New York, NY 10027

E-mail address: cm@math.columbia.edu 